\title{
The detection of real and apparent concomitant rotation in a three-dimensional cube: Implications for perceptual interactions
}

\author{
MARY A. PETERSON and GARY CHON-WEN SHYI \\ State University of New York at Stony Brook, Stony Brook, New York
}

\begin{abstract}
Apparent concomitant rotation, such as that seen when viewers move laterally relative to a depth-reversed three-dimensional wire cube, has been taken as evidence that perceived distance must precede perceived motion, a proposal that is consistent with taking-into-account theories. Another proposal has been that apparent concomitant motion arises from attention to proximal motion and, hence, cannot support general claims about motion perception. In two experiments, moving viewers saw 700-msec exposures of a cube biased to be seen in either veridical or reversed depth. The cube either rotated or remained stationary. Viewers rated the perceived rotation, using a 4-point scale. The results suggested that depth cues interact with other variables before perceived rotation is computed. Surprisingly, rotations that included a substantial apparent concomitant motion component were rated higher than equivalent rotations that included no illusory motion. This asymmetry suggests that taking-into-account theories must be amended.
\end{abstract}

When viewers move relative to stationary objects in depth, the difference in the angular velocity with which those objects displace on the retina is a function of the relative distances to those objects. It is assumed that this "motion parallax" normally goes unseen, providing the viewer with relative depth information (Miller, 1962; Rogers \& Graham, 1979).

In some situations, however, moving viewers report seeing an illusion that stationary objects at different distances are in relative motion; this motion has been called apparent concomitant motion (Gogel, 1979, 1980, 1982; Gogel \& Tietz, 1973, 1977). Gogel presents compelling evidence that apparent concomitant motion is reported whenever errors in perceived distance occur, regardless of the means through which the misperception of distance occurs (e.g., errors can be induced through fixation [Gogel, 1979; Gogel \& Tietz, 1977], binocular disparity [Gogel, 1980], or perspective cues [Gogel \& Tietz, $1974])$. For this reason, Gogel $(1979,1980,1982)$ has proposed that the perception of depth precedes the perception of motion (cf. Epstein, 1973) and that, at least in these circumstances, no perception of motion is possible without the prior perception of depth.

Gogel (e.g., 1982) has summarized these predictions in a perceptual equation in which the magnitude of the apparent concomitant motion is predicted to be a function of the actual distance to the object, the perceived distance, the extent of viewer movement, and the distance

This research was supported by a Public Health Service Award (2S07RR0706719) to the first author. The authors thank J. Hochberg and two anonymous reviewers for careful critiques of previous versions of this article, and R. Reeder, H. Tepper, and the late A. Castaldi for technical assistance. The first author is now at the Department of Psychology, The University of Arizona, Tucson, AZ 85721. to the point around which the line of sight to the object pivots. (This last variable allows Gogel to account for situations in which the object actually moves as the viewer moves.) The predictions made by Gogel's equation tend to be consistent with those made by taking-into-account theories in which apparent concomitant motion is explained as the best-fit solution for the perceived distance and the obtained parallax (Epstein, 1973; Hay \& Sawyer, 1969; Hochberg, 1981; Wallach, Stanton, \& Becker, 1974). In particular, taking-into-account theories predict that the velocity of the perceived motion (here called the theoretical motion, $\left.v^{\prime}\right)$ is that which would result in the obtained proximal displacement $(P)$ after taking into account the expected parallax given the perceived distance $\left(X \mathrm{e} \mid D^{\prime}\right)$ :

$$
v^{\prime}=P-\left(X \mathrm{e} \mid D^{\prime}\right) .
$$

Thus, taking-into-account theories also assume the priority of perceived distance, because the parallax expected for the perceived distance enters into the calculation of the perceived motion.

There has been some reticence in accepting the general claim that perceived distance precedes perceived motion. One reason is philosophical: The proposal concerning the priority of perceived distance for perceived motion implies perceptual causation, a claim that psychologists have long sought to avoid (Epstein, 1982; Hochberg, 1968, 1981). Apparent concomitant motion is one member of a class of "perceptually coupled" variables (Hochberg, $1956,1974)$, variables that normally covary in stimulation but that also covary perceptually. That is, even without changes in the physical array, viewers who are led to perceive a change in one variable of a perceptually coupled pair will perceive a concomitant change in the other. For example, relative distance and parallax nor- 
mally covary; moving viewers who perceive an illusory difference in the relative distance to two objects (e.g., two regions of a stereogram) also perceive apparent concomitant motion because the expected parallax is not obtained. This illusory motion is seen even in situations (such as that involving a stereogram) in which no relative motion occurs between near and far objects. Despite the fact that the possibility of perceptual causation must be confronted in the perceptual couplings, their study may reveal evidence concerning perceptual computations (see Epstein, 1982). Therefore, answers to questions regarding the ontological status of the variables that are weighed in the computations are important to perceptual theory.

Another important question is whether differential attention to some subset of the variables that interact to yield the apparent concomitant motion can affect their weight in the perceptual computations. Shebilske and Proffitt $(1981,1983)$ claim that viewers report apparent concomitant motion when they pay undue attention to the proximal motion at the expense of other motion attributes that might identify the apparent concomitant motion as illusory. For example, Shebilske and Proffitt point out that the path of the illusory motion must be more complex than a real motion path because apparent concomitant motion results from the addition of real motion vectors and apparent motion vectors. Thus, observers attending to the complexity of the motion path rather than to the proximal motion might be less likely to report apparent concomitant motion. If this were the case, the claim that perceived distance precedes perceived motion would not generalize to situations in which attention is not directed to the proximal motion and, therefore, would be of limited usefulness.

That apparent concomitant motion can be seen (1) between parts of a stereogram in which no relative motion occurs (Jerison, 1967; Peterson, 1986) and (2) in the direction opposite to the retinal motion (Gogel, 1982, 1983) argues against the possibility that viewers are attending to the proximal motion. In previous work, however, the magnitude of the apparent concomitant motion has increased as retinal motion increased (Shebilske \& Proffitt, 1983; but see Peterson, 1984), leaving open the possibility that viewers respond to the magnitude of the retinal motion and label rotation direction in some postperceptual stage capable of taking depth cues into account (Hochberg \& Peterson, 1987; Peterson, 1986). Therefore, it is important to pursue further the question of whether reports of apparent concomitant motion are related to the magnitude of the proximal motion.

There is little experimental work, other than Gogel's, that has attempted to identify the variables that interact to yield the apparent concomitant motion. And Gogel's experimental tests have tended to use indirect methods, making their interpretation somewhat difficult. For example, in most of his research, Gogel manipulated perceived distance (while holding constant actual distance, pivot distance, and extent of viewer movement) and measured the apparent concomitant motion. Typically, in a procedure such as this, the predicted and obtained magnitudes of the apparent concomitant motion would be compared. However, this was not done, perhaps because of the difficulties of manipulating perceived distance in the reduced experimental conditions that have been used (i.e., the stimulus has been a single light [Gogel, 1982] or a luminous line [Gogel, 1980] in a dark room; but see Gogel, 1979). Instead, Gogel and his colleagues used the reports about apparent concomitant motion as indirect measures of the perceived distance, and then compared the perceived distance (so obtained) with the manipulated distance. Although perceived distance tended to change in the direction predicted by the distance manipulations, the two were rarely identical. These discrepancies may be due to uncontrolled variables that contributed to the perception of distance (e.g., a tendency to see stimuli in reduced conditions as located at approximately $2 \mathrm{~m}$ distance; Gogel \& Tietz, 1973). Alternatively, the differences may imply that additional variables are weighed in the computation of the perceived motion (e.g., see Post \& Leibowitz, 1982), so that substituting measures of perceived motion into Gogel's equations cannot provide precise measures of perceived distance. Indirect measures do not allow these possibilities to be separated. More direct measures of the apparent concomitant motion might provide evidence regarding the need to supplement takinginto-account equations with other variables.

In the experiments reported here, we addressed some of these questions regarding apparent concomitant motion, using a three-dimensional (3-D) wire cube in a context-rich environment in which other objects were visible against a textured background. The wire cube was biased so as to be seen in reversed depth on some trials (back-biased trials) and in veridical depth on others (frontbiased trials), as shown in Figures 1A and 1B. When viewers make small lateral excursions relative to stationary depth-reversed objects, apparent concomitant motion is perceived: the object appears to rotate to follow the viewers (Mach, 1895/1959; Wallach et al., 1974).

The cube was stationary on one-third of the trials; on the rest of the trials, the cube rotated in either a clockwise or a counterclockwise direction. When the cube rotated, it did so at one of a number of speeds. Viewers were first instructed on parallax and then were asked to judge whether the cube was truly rotating or was truly stationary on each trial.

For any trial, the real rotation was known and the proximal motion and theoretical rotations could be estimated. When viewers make small lateral excursions, as they did in these experiments, the relative parallactic displacement between the front and rear faces of the cube approximates the relative displacements that would occur with rotation (Cutting, 1986, p. 188; Gogel \& Tietz, 1974; Wallach et al., 1974). Accordingly, proximal motion $(P)$ was estimated as the difference between the real rotation $(R)$ and the actual parallax $(\mathrm{Xa})$ :

$$
P=R-X \mathbf{a} .
$$


A positive sign was assigned to the parallax whenever the cube's true front face displaced to the left relative to the cube's back face as the viewer moved from left to right. Real rotations were signed in the same manner as the parallax: Rotations in which the front face displaced to the left relative to the back face (clockwise rotations) were positive; rotations in which the front face displaced to the right relative to the back face (counterclockwise rotations) were negative. Estimates of the proximal motion for each of the two conditions of parallax used in these experiments are shown in Table 1.

Theoretical rotations predicted by a taking-into-account theory were estimated for the front-biased and back-biased conditions using Equation 1. Signs were assigned to the expected parallax in the same manner as they were to the actual parallax. The actual parallax was taken as the expected parallax in the front-biased condition $(X e \mid f=X a)$. The complement of the actual parallax was taken as the expected parallax in the back-biased condition $(\mathrm{Xe} \mid \mathrm{b}=$ $-X a)$ because the true back face (perceived as the front face) would be expected to displace to the left relative to the true front face (perceived as the back face) as the viewer moved from left to right (see Figure 1C). The computed values of theoretical rotation are also shown in Table 1. Note that the theoretical rotation predicted in the back-biased condition is reversed. It has long been known that depth reversals are accompanied by reversals in the perceived direction of rotation (Sinsteden, 1860; von Hornbostel, 1922).

In addition, inasmuch as $X \mathrm{e}=-X \mathrm{a}$ in the back-biased condition, the $v^{\prime} s$ in the front-biased and back-biased stationary conditions differ by $-2 X \mathrm{X}$ before a reversed rotation direction is assigned to the back-biased cubes. This value, $-2 X a$, is the predicted velocity of the apparent concomitant rotation in a stationary depth-reversed cube (see Wallach et al., 1974). The apparent concomitant rotation is the sole source of theoretical rotation in some situations (e.g., when the back-biased cube is really stationary); it contributes to the theoretical rotation in other situations; and, in some situations (e.g., whenever the theoretical rotation is predicted to be zero in the backbiased condition), it is expected to cancel the real rotation. Therefore, on some trials (i.e., back-biased stationary trials), only apparent concomitant motion was expected to be present. On other trials (i.e., front-biased trials), only real motion was present. And on some trials, real motion and apparent concomitant motion were both expected to be present.

Viewers used a 4-point scale to rate whether the cube was really moving on each trial. If viewers can distinguish between apparent concomitant motions and real rotation under these conditions, their reports of rotation will vary with the real rotation and not with the proximal motion. Furthermore, their rotation reports should not vary with bias condition. If viewers respond to the theoretical rotation, however, even in this situation, where ample distance cues are provided by the context, their rotation ratings will be correlated with the theoretical rotations rather than with the real rotations or with the proximal motions. Furthermore, their ratings should vary with bias condition, inasmuch as the theoretical rotations for a given real rotation differ in both direction and magnitude as a function of bias condition.

We asked viewers to rate the perceived rotation rather than to null it (cf. Gogel, 1982) so that the task did not direct viewers' attention to any particular motion

Table 1

Estimated Values of Proximal Motion and Theoretical Rotations for Each Real Rotation for Each Parallax Condition in Experiments 1 and 2

\begin{tabular}{|c|c|c|c|c|c|c|c|}
\hline \multicolumn{4}{|c|}{ 14'/sec Parallax } & \multicolumn{4}{|c|}{ 27'/sec Parallax } \\
\hline \multirow[b]{2}{*}{ Real } & \multirow[b]{2}{*}{ Proximal } & \multicolumn{2}{|c|}{ Theoretical } & \multirow[b]{2}{*}{ Real } & \multirow[b]{2}{*}{ Proximal } & \multicolumn{2}{|c|}{ Theoretical } \\
\hline & & $\begin{array}{c}\text { Back } \\
(X e=-14)\end{array}$ & $\begin{array}{c}\text { Front } \\
(X e=14)\end{array}$ & & & $\begin{array}{c}\text { Back } \\
(X e=-27)\end{array}$ & $\begin{array}{c}\text { Front } \\
(\mathrm{Xe}=27)\end{array}$ \\
\hline \multicolumn{8}{|c|}{ Experiment 1} \\
\hline $\begin{array}{r}+28 \\
+14 \\
0 \\
-14 \\
-28\end{array}$ & $\begin{array}{r}+42 \\
+28 \\
+14 \\
0 \\
-14\end{array}$ & $\begin{array}{r}-56 \\
-42 \\
-28 \\
-14 \\
0\end{array}$ & $\begin{array}{r}+28 \\
+14 \\
0 \\
-14 \\
-28\end{array}$ & $\begin{array}{r}+28 \\
+14 \\
0 \\
-14 \\
-28\end{array}$ & $\begin{array}{r}+56 \\
+42 \\
+28 \\
+14 \\
0\end{array}$ & $\begin{array}{l}-84 \\
-70 \\
-56 \\
-42 \\
-28\end{array}$ & $\begin{array}{r}+28 \\
+14 \\
0 \\
-14 \\
-28\end{array}$ \\
\hline \multicolumn{8}{|c|}{ Experiment 2} \\
\hline $\begin{array}{r}+14 \\
0 \\
-14 \\
-28 \\
-42\end{array}$ & $\begin{array}{r}+28 \\
+14 \\
0 \\
-14 \\
-28\end{array}$ & $\begin{array}{r}-42 \\
-28 \\
-14 \\
0 \\
+14\end{array}$ & $\begin{array}{r}+14 \\
0 \\
-14 \\
-28 \\
-42\end{array}$ & $\begin{array}{r}+28 \\
+14 \\
0 \\
-14 \\
-28 \\
-42 \\
-56 \\
-70 \\
-84\end{array}$ & $\begin{array}{r}+56 \\
+42 \\
+28 \\
+14 \\
0 \\
-14 \\
-28 \\
-42 \\
-56\end{array}$ & $\begin{array}{r}-84 \\
-70 \\
-56 \\
-42 \\
-28 \\
-14 \\
0 \\
+14 \\
+28\end{array}$ & $\begin{array}{r}+28 \\
+14 \\
0 \\
-14 \\
-28 \\
-42 \\
-56 \\
-70 \\
-84\end{array}$ \\
\hline
\end{tabular}

Note-Motions in which the true front face displaces to the left relative to the true back face are assigned positive signs; those in which the true front face displaces to the right relative to the true back face are assigned negative signs. 
parameter. Thus, viewers were free to attend to whichever motion attributes might allow them to distinguish between real rotation and apparent concomitant rotation. Because of the use of a rating procedure, these experiments do not permit precise comparison of the predicted and obtained magnitudes of the theoretical rotation. Wherever possible, however, we will examine whether the predicted and obtained magnitudes are the same. For example, if the rotation ratings suggest that real and apparent concomitant motion cancel in conditions in which the theoretical rotation is predicted to be zero, that will suggest a strong similarity between the predicted and the perceived magnitudes of theoretical rotation in those conditions.

\section{EXPERIMENT 1}

\section{Method}

Subjects. Four women enrolled in summer courses at the State University of New York at Stony Brook were paid to participate in this experiment. All had vision that was normal or corrected to normal.

Stimulus and Apparatus. The stimulus was a 3-D cube, $15 \mathrm{~cm}$ on a side, constructed of black wire rods, $1.8 \mathrm{~mm}$ thick. It was mounted on a variable-speed stepper motor controlled by a Rogers Prime Mover System in an Apple Ile. The cube was attached to the motor by a vertical wire extending from its lower left corner, which formed the axis about which the cube rotated. Both this vertical mounting wire and the apparatus driving the cube were hidden from view by a tan-colored textured cloth that also provided a foreground and a background against which the cube was viewed.

The cube was located $230 \mathrm{~cm}$ from the subject and $11 \mathrm{~cm}$ below eye level. At this distance, each face subtended $3.7^{\circ}$ of visual angle. The cube was positioned so that the lower left face was the back face, as shown in Figure 1. Bias toward either a veridical or a reversed depth organization was achieved by thickening the wires composing either the front or the back face with $5-\mathrm{mm}$ matte black soda straws. This cue has served as a reliable means for manipulating the perceived relative depth in a luminous line (Gogel \& Tietz, 1974) and in a moving cube (Hochberg \& Spiron, 1985).

The room was well lighted. In addition, the cube was back-lighted by reflections from four hooded $150-\mathrm{W}$ bulbs that faced away from the viewers and reflected light off the tan cloth back onto the cube. The hoods shielding these lights were within the subject's visual field and served as a stationary frame of reference. The visual field was about $155^{\circ}$ in diameter. A second cube, identical in size to the experimental cube, but nonreversible, was placed $141 \mathrm{~cm}$ from the viewers; its top was $2 \mathrm{~cm}$ below the bottom of the experimental cube. Thus, observers viewed the experimental cube with other stationary unambiguous objects within their visual fields.

A viewing apparatus controlled the observer's rate of movement and the duration of cube exposure. The viewing apparatus, hung like a pendulum from an overhead railing, was moved manually by the experimenter to the beat of a metronome at one of two rates: It was moved around its pivot either $11^{\circ}$ at $.75 \mathrm{~Hz}$ or $17.25^{\circ}$ at $.93 \mathrm{~Hz}$. These rates of movement resulted in approximately $14^{\prime} / \mathrm{sec}$ and $27 \%$ sec parallax between the front and rear faces of the cube.

Procedure. The subjects participated in the experiment individually. They were told that we were interested in their ability to determine whether a 3-D cube rotated or remained stationary as they moved. They were instructed on apparent concomitant motion, and allowed first to view stationary objects at different distances in the room and then to view the front and rear faces of the (unbiased) cube as they moved laterally. The subjects were told that this was not real rotation, and that they were not to label it as such during the experiment. Next, the subjects viewed the cube as it oscillated between clockwise and counterclockwise rotation for $8 \mathrm{sec}(2 \mathrm{sec}$ per half cycle), and they labeled the direction of rotation.

An electronic shutter (Gerbrands G1166S) and nosepiece were positioned on the viewing apparatus for each subject's left eye. Viewing was monocular; the subject either covered her right eye with an eyepatch or simply closed that eye during the experimental sequences. Before the experiment began, the subjects practiced moving behind the viewing apparatus so that they could anticipate their extent, direction, and speed of movement during the experimental trials. They voluntarily kept pace with the viewing apparatus by grasping a handle on it and by synchronizing their motion with the beat of the metronome. Thus, subject motion, although not free, was not passive.

Each trial began with three half cycles of subject motion with the shutter closed. During this time, the cube was rotated to one of five predetermined starting positions and the rotation sequence (if any) was initiated. These five starting positions for the cube were used so that the viewers would not infer rotation from a change in the cube's position. Regardless of starting position, the back face of the cube was always to the left of the front face, as shown in Figure $1 \mathrm{~A}$, and remained in that relative position throughout the rotation.

The shutter opened for $700 \mathrm{msec}$ in the middle of the subject's fourth half-cycle of movement. Thus, the subject was always moving from left to right when viewing the cube and the actual parallax was always positive (i.e., the front face of the cube displaced to the left relative to the back face). The short exposure duration served two functions. First, it allowed us to keep the viewer's motion approximately synchronized with the real motion. Second, with such brief exposures, both the real rotation vectors and the parallax were approximately linear, and we were therefore better able to approximate their sum by addition.

The subjects participated in two to four practice trials. Half of the subjects practiced with back-biased cubes; the other half practiced with front-biased cubes. These practice trials were followed by 120 experimental trials. Half of the subjects began by viewing the cube as they moved at the $27^{\prime} / \mathrm{sec}$ rate; the other half began by moving at the $14^{\prime} / \mathrm{sec}$ rate. (The subjects practiced moving at the new rate when parallax conditions switched.)

Half of the subjects began by viewing a back-biased cube; the other half began by viewing a front-biased cube. Trials were run in blocks of 10 . Biasing was varied across blocks in a counterbalanced order, unobserved by the subject. For each parallax condition, 30 motion sequences were presented: 10 stationary, 10 clockwise, and 10 counterclockwise. On half of the rotating sequences, the cube rotated at $2.5^{\circ} / \mathrm{sec}$, creating $14^{\prime} / \mathrm{sec}$ angular velocity between the front and rear faces. On the other half, the cube rotated at $5.0 \% / \mathrm{sec}$, creating $28 \% \mathrm{sec}$ angular velocity between the cube's two faces. Motion sequences were presented randomly within blocks, with the stipulation that each subject view each sequence once in each condition of bias for each parallax.

To report perceived rotation, the subjects used a 4-point rating scale (McNicol, 1972, p. 103) as follows: $1=$ certain the cube was stationary; 2 = uncertain, guess stationary; 3 = uncertain, guess rotating; and $4=$ certain that the cube was rotating. When they reported " 3 " or " 4 ," the subjects also reported whether the perceived direction of rotation was clockwise or counterclockwise.

The subjects were told that the cube would rotate on only half of the trials, and that it would rotate at two different speeds. They were asked to try to see the face formed by the thicker wires as the front face and to report whenever it did not appear to be in front. (On only one occasion, and that was in the back-biased condition, did one subject report that the thicker-edged face appeared behind; her response on this trial was not analyzed.) The subjects were asked to fixate the center of the thicker-edged face during their brief view of the cube. 


\section{Results}

As shown in the top half of Figure 2, mean rotation ratings were lower in the front-biased condition than in the back-biased condition $[F(1,3)=21.98, p<.02]$. In addition, rotation ratings for clockwise motion were higher than for counterclockwise rotation $[F(1,3)=44.4$, $p<.007]$. An interaction between bias and speed showed that motion ratings increased as speed increased in the front-biased condition but not in the back-biased condition, where motion ratings either remained the same or
A

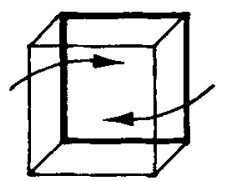

B

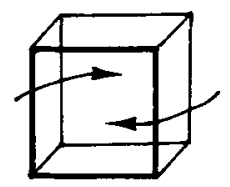

C

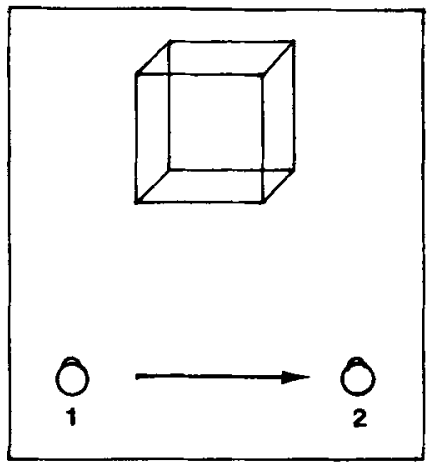

Front-Biased

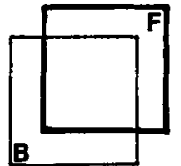

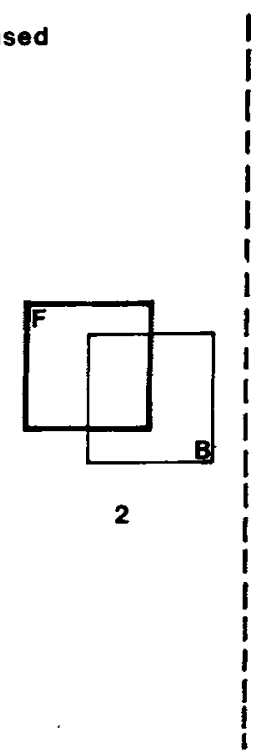

$x_{e \mid f}=x_{a}$

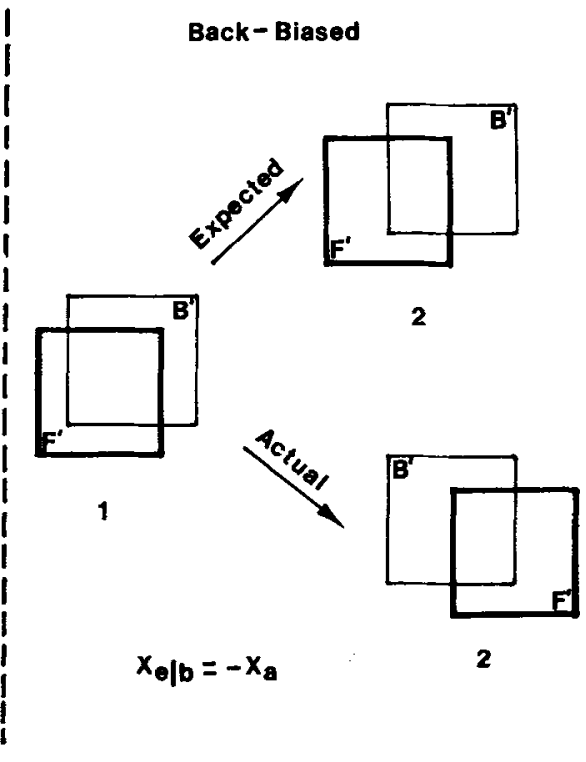

Figure 1. $A$ and $B$ are drawings of the 3-D cube used in Experiments 1 and 2. The lower left face of the cube was always the back face. $A$ = front-biased condition; $B=$ back-biased condition. The arrows in $A$ show the relative motions of the front and the rear faces when the cube rotates in a clockwise direction. Those vectors specify counterclockwise rotation when the back face appears forward, as in B. In C, 1 and 2 represent the viewer's position at the beginning and end of the observation interval. As the viewer moves from 1 to 2 , the near face of the cube displaces to the left relative to the far face at a rate that is a function of the viewer's movement (and fixation). For the front-biased stationary condition, shown in the bottom left panel, we assumed that the expected parallax was the actual parallax $(X \mathrm{X} \mid \mathrm{f}=\mathrm{Xa})$. For the back-biased stationary condition, shown in the bottom right panel, the expected parallax was the complement of the actual parallax $(X \mathbf{e} \mid \mathbf{b}=-X \mathbf{a})$. 


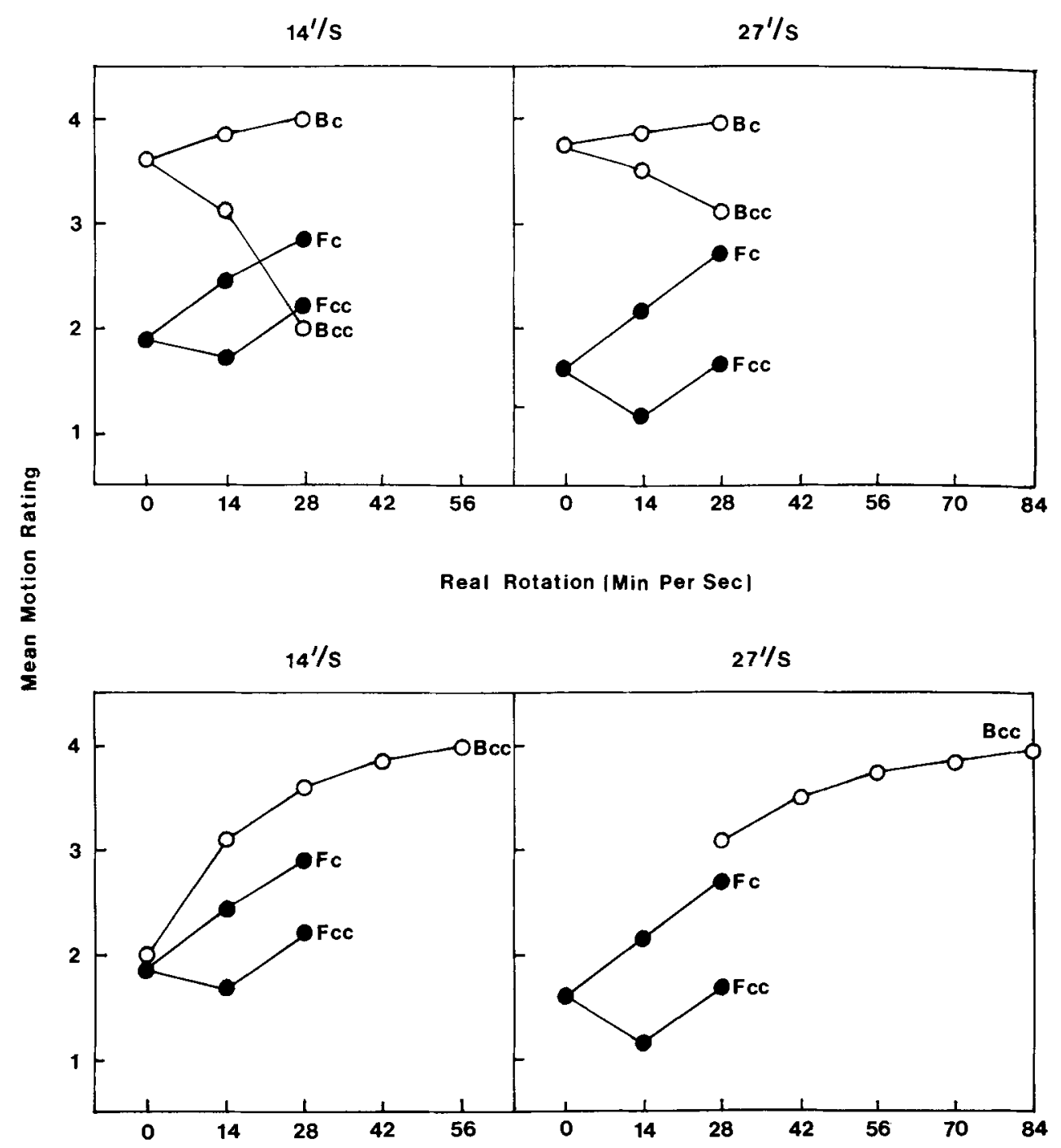

Theoretical Rotation (Min Per Sec)

Figure 2. Rotation ratings in Experiment 1. The top panel shows the ratings as a function of the real rotation. The 14'/sec parallax condition is shown in the left panel; the 27'/sec parallax condition is shown in the right panel. $\mathrm{F}=$ front-biased, $\mathrm{B}=$ back-biased, $\mathrm{c}=$ clockwise, and $\mathrm{cc}=$ counterclockwise. The bottom panel shows the same data as a function of theoretical rotation.

decreased as speed increased $[F(1,3)=102.4$, $p<.002$ ]

Ratings in the back-biased condition gave no sign that viewers were able to distinguish between real rotation and theoretical rotation. Two comparisons were made in this regard. First, the ratings given to the stationary and moving back-biased cube were compared: On back-biased trials in the 27 ' $\mathrm{sec}$ parallax condition, the mean ratings for the stationary cubes in which apparent concomitant rotation was expected to occur (3.75) did not differ from the mean ratings assigned to the rotating cubes in which apparent concomitant rotation added to the real rotation (3.6) $[t(3)=1.64, p>.1]$; in the $14^{\prime} / \mathrm{sec}$ parallax con- dition, the mean ratings were higher for the stationary cube (3.62) than for the rotating cube (3.2) $[t(3)=4.19$, $p<.05]$. Second, the ratings given to the apparent concomitant rotation in the stationary back-biased cube were compared with the ratings given to the rotating frontbiased cube: The apparent concomitant rotation in the back-biased cube was rated higher than the real rotation seen without an illusory component in the front-biased cube $\left[t \mathrm{~s}(3)=3.37\right.$ and 6.8 in the $14^{\prime} / \mathrm{sec}$ and $27^{\prime} / \mathrm{sec}$ parallax conditions, respectively].

Moreover, the subjects were not simply responding to the proximal motions: Pearson product moment correlations between subjects' ratings and the magnitude of the 
proximal motions were low, as shown in Table $2 .{ }^{1}$ Correlations between viewers' rotation ratings and the magnitude of the theoretical rotations were high, however (see Table 2). ${ }^{2}$

The rotation ratings were replotted as a function of the theoretical rotations in the bottom half of Figure 2, where it is apparent that the motion ratings increased with the theoretical rotations. In addition, the results suggest that the real rotation adds to the apparent concomitant rotation. Consider the case of a cube moving at $28^{\prime} / \mathrm{sec}$ counterclockwise in the $14^{\prime} / \mathrm{sec}$ parallax condition. As can be seen in Table 1 , this real rotation yields a theoretical rotation of zero in the back-biased condition. That is, the real rotation should be cancelled by the apparent concomitant rotation if the two are adding perceptually. As shown in Figure 2, the mean rotation rating of 2.0 shows that this is, indeed, what viewers perceived. Both the backbiased condition, in which the rotating cube approximately cancels the apparent concomitant rotation, and the frontbiased stationary cube receive mean ratings of approximately 2.0 .

A rather surprising finding was that viewers rated rotations that resulted in approximately zero proximal motion as stationary in the front-biased condition and as moving in the back-biased condition. In addition, theoretical rotations less than or equal to the parallax were rated as stationary in the front-biased condition, but not in the back-biased condition, at least not in the $14^{\prime} / \mathrm{sec}$ parallax condition, in which theoretical rotations of these magnitudes could be examined. Overall, viewers tended to be more confident that they were seeing motion in the backbiased condition rather than the front-biased condition: Most of the ratings for the front-biased condition fall below 3.0.

\section{Discussion}

The results of Experiment 1 show that viewers who report apparent concomitant motion in these conditions are not attending to the magnitude of the proximal motion and are not labeling rotation direction in some postperceptual process that takes depth into account. Correlations between the rotation reports and the proximal motion were low and nonsignificant. Thus, Experiment 1 suggests that attention to the proximal motion is not responsible for reports of apparent concomitant motion.

In addition, our results suggest that viewers are not rejecting the apparent concomitant motion on the basis of path complexity. The apparent concomitant-motion component of the perceived rotation is nonrigid (see Ullman, 1979, p. 182), whereas the real rotation is rigid. Nevertheless, apparent concomitant rotation was rated higher than the real rotation. Furthermore, regardless of bias condition, rotation reports were correlated with the theoretical estimates of rotation rather than with the real rotation.

The real rotation apparently adds with the apparent concomitant rotation, as evidenced by the low ratings assigned to the rotation sequences that yielded approximately zero theoretical rotation in the $14^{\prime} / \mathrm{sec}$ parallax condition. (Un- fortunately, this last finding was not replicable in the 27 /sec parallax condition, because, in this experiment, the real rotations used in the two parallax conditions were equated but the theoretical rotations were not. This was corrected in Experiment 2.) Thus, the results of Experiment 1 extend Gogel's (1982) evidence regarding the additivity of real and apparent concomitant motion in a spot of light to a situation involving a real 3-D object in a rich environment.

There were some unexpected findings, however. First, clockwise real rotations were rated higher than counterclockwise real rotations. This is consistent with Wallach et al.'s (1974) data, which, although unremarked upon in the report, suggest that a viewer is less sensitive to rotations that occur in the same direction as his/her own movement (corresponding to counterclockwise rotations here) than to rotations that occur in the opposite direction (here, clockwise rotations). Another possibility is that counterclockwise rotations resulted in higher velocity theoretical rotations in the back-biased condition, thereby accounting for the difference between the clockwise and counterclockwise real rotations. However, this explanation cannot account for the difference between the clockwise and counterclockwise rotations in the front-biased condition (although it should be noted that both clockwise and counterclockwise rotations received ratings less than 3.0 in the front-biased condition). (We explored the difference between clockwise and counterclockwise rotation further in Experiment 2, where we equated the theoretical rotations rather than the real rotations.)

A second rather surprising finding was that viewers were less likely overall to report motion in the front-biased condition than in the back-biased condition. Of particular interest was the finding that theoretical rotations with magnitudes less than or equal to the parallax were rated as stationary in the front-biased condition but as rotating in those cases that could be examined in the back-biased condition.

One possible explanation for the difference between the front- and back-biased conditions may be that the real rotation was below some threshold. As can be seen in Table 1, the magnitudes of the theoretical rotations in the front-biased condition were generally less than those in the back-biased condition. Wallach et al. (1974) have shown that small rotations escape detection by observers who are moving, and indeed, the mean rating for all the real-rotation sequences in the front-biased condition was 2.0. If the real rotation was below some threshold, so that only the apparent concomitant rotation was seen on many trials, subjects (who had been led to expect real rotation to occur on half of the trials) may have been willing to apply a rotation label to the rotation seen in the backbiased condition only because there was no real rotation with which to compare it. If so, we cannot be sure whether apparent concomitant motion is distinguishable from real rotation when the latter is above threshold.

Accordingly, in the next experiment, we used a larger range of real rotations so that suprathreshold real rotations would be available for comparison with the theo- 
retical rotations in the back-biased condition. Second, rather than matching the magnitudes of the real rotations in the clockwise and counterclockwise directions, we matched the magnitudes of the theoretical rotations in the front- and back-biased conditions. Third, we gave subjects no instructions about how often to expect real rotation. These conditions should serve as a sensitive test of whether viewers can distinguish real rotation from apparent concomitant rotation or from rotation that includes a substantial apparent concomitant motion component.

\section{EXPERIMENT 2}

\section{Method}

Subjects. Nine students at the State University of New York at Stony Brook participated in this experiment in order to fulfill a course requirement. All had vision that was normal or corrected to normal.

Stimulus and Apparatus. The stimulus and apparatus were those used in Experiment 1. There were two parallax conditions: 14'/sec and $27^{\prime} / \mathrm{sec}$. The real rotations used are shown in the bottom half of Table 1. A wide range of counterclockwise rotations was used to provide suprathreshold real rotation. Clockwise rotations were included so that the magnitudes of the predicted theoretical rotations would be equivalent to the magnitudes of the real rotations.

Procedure. The instructions were the same as those used in Experiment 1 , except that the subjects received no instructions about how often to expect to see real motion. The subjects participated in four practice trials ( 5 practiced with a back-biased cube, and 4 practiced with a front-biased cube). These practice trials were followed by 56 experimental trials-36 at $27^{\prime} / \mathrm{sec}$ parallax and 20 at $14 ' / \mathrm{sec}$ parallax. The subjects viewed each real-rotation sequence twice in each condition of bias and parallax. Half began by moving at the $14^{\prime} / \mathrm{sec}$ rate; the other half moved at the $27^{\prime} / \mathrm{sec}$ rate. Half began by viewing a back-biased cube; the other half viewed a front-biased cube. Trials were run in blocks of 9 in the $27 \%$ sec parallax condition and in blocks of 10 in the 14 '/ $\mathrm{sec}$ parallax condition. The bias of the cube was counterbalanced across blocks. Trials on which subjects reported that the thicker-edged face of the cube appeared to be behind were repeated later. (Such reports occurred on only four trials across all subjects.)

\section{Results}

The subjects reported rotation on $100 \%$ of the stationary back-biased trials in which apparent concomitant rotation was expected to occur. As expected (see Table 1), the rotation direction reported on all of these trials was counterclockwise. The ratings for the apparent concomitant rotation were not lower than those for the real rotation.

As shown in Table 2, correlations between the subjects' mean rotation ratings and the proximal and theoretical motions show that the magnitude of the motion ratings varied only with the magnitude of the theoretical rotations. No differences were obtained between the clockwise and counterclockwise real rotations.

As can be seen in the top half of Figure 3, as real rotation increased, mean rotation ratings increased in the front-biased condition, but decreased in the back-biased condition $\left[F \mathrm{~s}(1,8)=20.95\right.$ and 23.73 for $14^{\prime} / \mathrm{sec}$ and $27^{\prime} / \mathrm{sec}$ parallax, respectively; $\left.p<.001\right]$. As shown in Table 1, however, in the back-biased condition, predicted
Table 2

Correlations Between Viewers' Ratings and Proximal Motions and Theoretical Rotations in Experiments 1 and 2

\begin{tabular}{ccccccc}
\hline & & \multicolumn{5}{c}{ Subjects } \\
\cline { 3 - 6 } Rotation & Parallax & P.S. & M.A. & E.P. & S.M. & Mean \\
\hline \multirow{5}{*}{ Proximal } & $14^{\prime} / \mathrm{sec}$ & .450 & .528 & .377 & .398 & \\
& $27^{\prime} / \mathrm{sec}$ & $.680^{*}$ & .230 & .277 & .313 & \\
Theoretical & $14^{\prime} / \mathrm{sec}$ & $.660^{*}$ & $.679^{*}$ & $.764 \dagger$ & $.738^{*}$ & \\
& $27^{\prime} / \mathrm{sec}$ & $.748 \dagger$ & $.746 \dagger$ & $.929 \dagger$ & $.876 \dagger$ & \\
& & Experiment 2 & & & \\
Proximal & $14^{\prime} / \mathrm{sec}$ & & & & & .051 \\
& $27^{\prime} / \mathrm{sec}$ & & & & & .007 \\
Theoretical & $14^{\prime} / \mathrm{sec}$ & & & & $.595^{*}$ \\
& $27^{\prime} / \mathrm{sec}$ & & & & $.707 \dagger$ \\
\hline${ }^{*} p<.05$. & $\dagger p .01$. & & &
\end{tabular}

theoretical rotations increased as real rotation decreased. When rotation ratings are plotted as a function of the theoretical rotations, as in the bottom half of Figure 3, it is clear that rotation ratings increased as a function of the predicted theoretical rotations in both conditions of bias. However, note that in the front-biased conditions, real rotations less than $14^{\prime} / \mathrm{sec}$ in the $14^{\prime} / \mathrm{sec}$ parallax condition and less than $28^{\prime} / \mathrm{sec}$ in the $27^{\prime} / \mathrm{sec}$ parallax condition received mean motion ratings lower than 2.5 , regardless of whether the rotation was clockwise or counterclockwise. In the back-biased condition, clockwise theoretical rotations in this range were given low ratings, but counterclockwise theoretical rotations in this range were always rated higher than $3.0 .^{3}$

Again, as in Experiment 1, front- and back-biased conditions differed in the rotation ratings assigned when the proximal motion was approximately zero (i.e., $14^{\prime} / \mathrm{sec}$ and $28^{\prime} / \mathrm{sec}$ counterclockwise rotation in the $14^{\prime} / \mathrm{sec}$ and $27^{\prime} / \mathrm{sec}$ parallax conditions, respectively). According to taking-into-account theories, rotations sufficient to cancel the expected parallax should be perceived whenever the proximal motion is zero. This should be true regardless of bias condition. Nevertheless, apparent concomitant rotation was seen in the back-biased condition, but real rotation was not seen in the front-biased condition. These differences were significant: Ratings for the $14^{\prime} / \mathrm{sec}$ parallax were 3.33 and 1.67 for the back-biased and frontbiased conditions; for the $27^{\prime}$ /sec parallax condition, they were 3.44 and $1.89[t s(8)=5.16$ and 4.6 , respectively].

\section{Discussion}

These results show that even when the predicted magnitudes of the theoretical rotations are equated in the frontand back-biased conditions, viewers cannot distinguish real rotation from apparent concomitant rotation or from theoretical rotations that include a substantial apparent concomitant motion component. In addition, the results support the proposal that real and apparent concomitant rotation add perceptually: Low ratings were assigned to back-biased cubes in which the predicted theoretical rotation was approximately zero, and ratings increased as the theoretical rotations resulting from the addition of real 


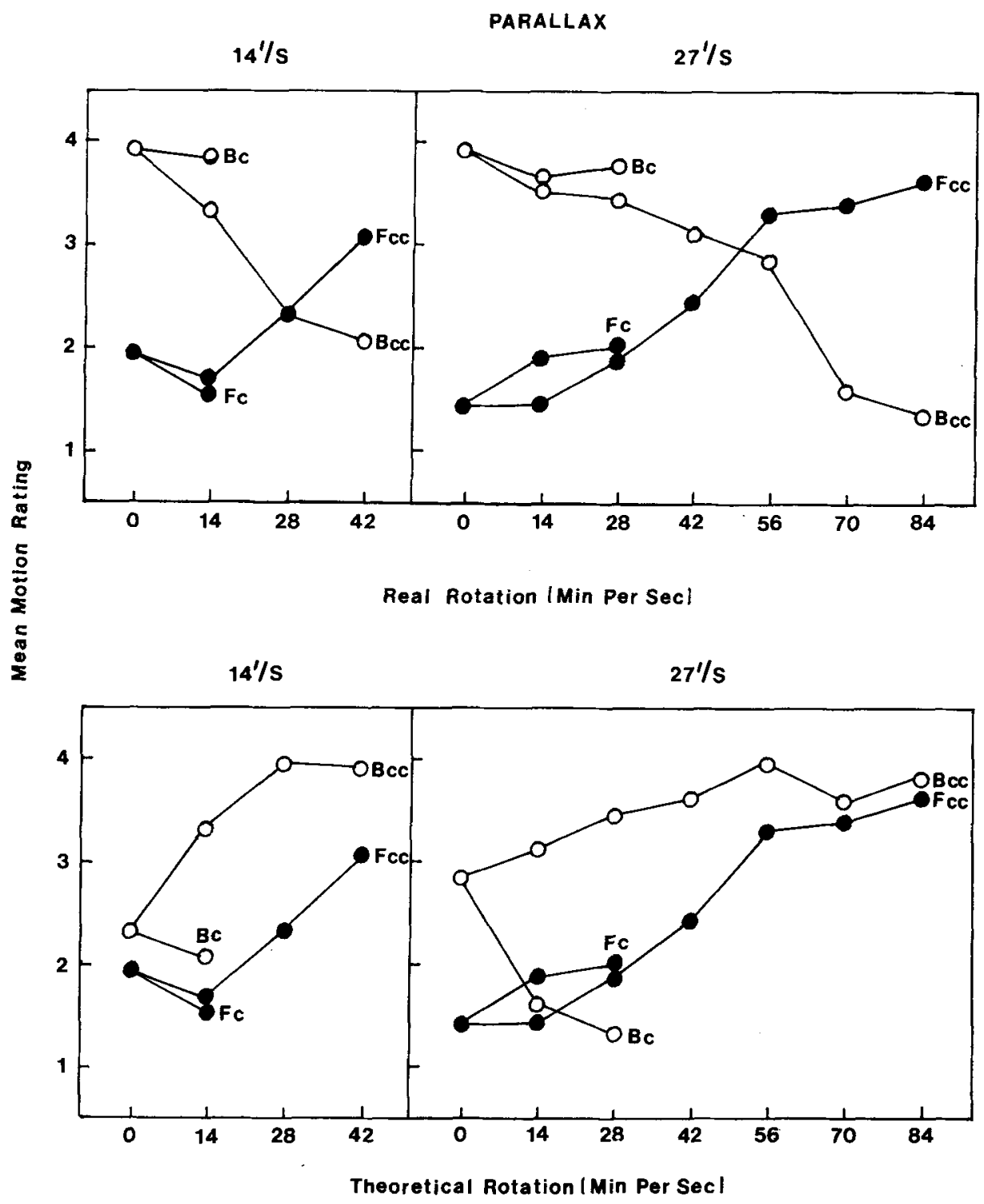

Figure 3. Rotation ratings in Experiment 2. The top panel shows the ratings as a function of the real rotation. The 14 '/sec parallax condition is shown in the left panel; the 27 '/sec parallax condition is shown in the right panel. $F=$ front-biased, $B=$ back-biased, $c=$ clockwise, and $\mathrm{ec}=$ counterclockwise. The bottom panel shows the same data as a function of theoretical rotation.

and apparent concomitant motion vectors increased. Furthermore, the results of Experiment 2 replicate those of Experiment 1 in showing that viewers reporting apparent concomitant motion were not attending to the proximal motion: Correlations between viewers' ratings and proximal motions were low.

In Experiment 2, as in Experiment 1, we again obtained significant differences between the front- and back-biased conditions. First, in the front-biased condition, the rotation ratings increased with the real rotation. In the backbiased condition, however, rotation ratings decreased as the real rotation increased. This difference between the bias conditions can be accounted for by the theoretical rotations, as shown in the bottom panel of Figure 3. Second, theoretical rotations of equal magnitude did not receive equal ratings in the front- and back-biased conditions. This difference was unexpected and suggests that the perceptual interactions that result in the perception of rotation in these situations may be more complex than predicted to date.

\section{GENERAL DISCUSSION}

The results of these two experiments show that the apparent concomitant motion that accompanies depth reversal in a wire cube does not result from attention to the 
proximal motion: Viewers' rotation ratings increased with the theoretical rotations rather than with the real rotations or the proximal motions. Indeed, correlations with the proximal motions were low and insignificant in both experiments. Instead, the results correspond broadly with what one would expect on the basis of a taking-intoaccount model, in which the perception of relative distance within the object is the basis for the computation of the apparent concomitant motion (Epstein, 1982; Gogel, 1982).

The differences that we obtained between the frontbiased and the back-biased conditions were not predicted by a taking-into-account model or by any of the alternate models, however. Particularly surprising is the fact that in the front-biased condition, neither clockwise nor counterclockwise real rotation with a magnitude less than or equal to the parallax were rated as motion, whereas in the back-biased condition, counterclockwise theoretical rotations in this range received high ratings, even though clockwise theoretical rotations of the same magnitudes received low ratings. These results were not predicted by Equation 1 . Nor can they be predicted easily by Gogel's perceptual equations. One way to account for these effects within Gogel's model would be to suppose that the perceived distance between the front and back faces of the depth-reversed cube increased dramatically as the counterclockwise theoretical rotation decreased from $28^{\prime} / \mathrm{sec}$ to $14^{\prime} / \mathrm{sec}$, but remained approximately the same as the clockwise theoretical rotations decreased from $28^{\prime} / \mathrm{sec}$ to $14^{\prime} / \mathrm{sec}$. Although we do not know precisely how reversal occurs (i.e., whether the front and rear faces switch apparent positions or whether one face flips around the other), it is unlikely that large variations in the perceived depth in the cube accompany these changes in perceived rotation. Thus, the discrepant ratings assigned to slow rotations in the back- and front-biased conditions suggest that other variables in addition to those considered to date (e.g., perceived distance, actual distance, pivot distance, and viewer movement) must be contributing to the perception of motion in this situation.

\section{Eye Movements}

One candidate for an additional variable to be considered has been suggested by Post and Leibowitz (1982), who propose that viewers who misperceive distance may make vergence errors which, in turn, may result in inaccurate compensatory eye movements when viewers move. Furthermore, they found that the direction of the pursuit movement that could correct for these inaccurate eye movements differed for viewers who observed a single light source when their eyes were under- or overconverged. The direction difference might account for the apparent concomitant motion.

This explanation cannot account for the differences between the front- and back-biased conditions obtained here, however. Although pursuit movements made to correct vergence-induced errors might add a translatory appar- ent concomitant motion component to the entire cube, they should not affect the relative apparent concomitant motion between the front and back faces of the cube. In addition, the cube really rotated on many trials, and rotation began from one of five different positions. Because of this, compensatory eye movements made to either the average location of the stationary cube or to the location seen in the initial view would be in error as often in the front-biased condition as in the back-biased condition. Consider a cube rotating counterclockwise at $42^{\prime} / \mathrm{sec}$ in the $27^{\prime} / \mathrm{sec}$ parallax condition. Rotation is seen in the backbiased condition and not in the front-biased condition. In order for the discrepancy between the location of the eye and the location of the face perceived to be the front face in the back-biased condition to equal that in the frontbiased condition, the size of the compensatory eye movement made in the back-biased condition would have to be twice as large as necessary. There is no evidence that vergence-induced errors of this magnitude occur.

\section{Complexity of the Motion Path}

Remember that the path of the theoretical motion in the back-biased cube must be complex (Shebilske \& Proffitt, 1981, 1983; Ullman, 1979). In particular, the front and back faces of the cube rotate at different speeds, yielding a nonrigid rotation in which local regions of the cube move relative to each other. Contrary to Shebilske and Proffitt, our observers did not use this information to reject the theoretical rotation in the back-biased condition. Instead, the difference between the ratings assigned to the clockwise and the counterclockwise theoretical rotations in the back-biased condition show that the larger the apparent concomitant motion component (and hence, the greater the nonrigidity), the more likely viewers are to rate the rotation as motion. Consider theoretical rotations of -14 and -28 in the back-biased $27^{\prime} / \mathrm{sec}$ parallax condition, corresponding to real rotations of -28 and -42 and proximal motions of 0 and -14 . The expected parallax $(-27)$ is larger than the proximal motion, so the predicted theoretical rotation is largely composed of apparent concomitant rotation. On the other hand, theoretical rotations of +14 and +28 correspond to real rotations of -70 and -84 and to proximal motions of -42 and -56 . The expected parallax $(-27)$ is less than the proximal motion, so the apparent concomitant motion component is cancelled in the predicted theoretical rotation. Ratings suggest that rotation is seen in the former condition, but not in the latter condition. Thus, it appears that the nonrigidities in the apparent concomitant rotation may actually contribute to the perception of rotation.

This proposal must be explored further. In particular, it will be important to know whether viewers can rate the rotation in the back-biased condition as nonrigid. Some rating research suggests that viewers are quite good at perceiving nonrigidity (Todd, 1982), although some types of small local distortions do not influence rigidity judgments (Cutting, 1987). Regardless of whether the nonri- 
gidities in the apparent concomitant rotation can be rated, they seem to exert an influence on the detection of rotation by moving observers.

In addition, the role of the 700 -msec exposure duration will be explored further. This short exposure duration was used so that viewers would not see that the direction of the apparent concomitant rotation reversed direction whenever they did. However, the exposure duration may have influenced perceived depth or perceived rotation.

\section{Priority of Perceived Distance}

As is apparent in the discussion above, our interpretation of the data assumes that the depth cues provided by the biasing are read before the apparent concomitant motion is seen. Thus, Gogel may be correct in assuming that depth cues are effective before the motion is computed. However, perceptual causation is not necessarily implied by the prior action of the depth cues. Depth cues may interact with other stimulus information at the stage where theoretical rotation is computed, but this need not imply that one perceptual variable causes another perceptual variable (see Oyama, 1977). Experiments, such as Epstein's (Epstein \& Broota, 1986; Epstein \& Lovitts, 1985), that explore the relative automaticity of the registration of the depth cues and the perception of the apparent concomitant motion would be interesting in this regard.

\section{Summary}

The present experiments provide strong evidence that apparent concomitant rotation does not result from attention to the proximal mode. Furthermore, the results of these experiments suggest that the models presented to date do not sufficiently account for the perceived rotation seen when moving observers view briefly exposed cubes. Although the results of the two experiments reported here fit the predictions from taking-into-account theories reasonably well, they strongly suggest that additional computations are necessary to explain the differences between the front- and back-biased conditions. In particular, the possibility that sensitivity to motion nonrigidities (or attention to motion nonrigidities) might account for the differences between the two conditions must be explored further.

\section{REFERENCES}

CutTing, J. E. (1986). Perception with an eye for motion. Cambridge, MA: MIT Press.

Cutring, J. E. (1987). Rigidity in cinema seen from the front row, side aisle. Joumal of Experimental Psychology: Human Perception \& Performance, 13, 323-334.

EPSTEIN, W. (1973). The process of "taking-into-account" in visual perception. Perception, 2, 267-285.

EpsteIn, W. (1982). Percept-percept couplings. Perception, 11, 75-88.

Epstein, W., BrootA, K. D. (1986). Automatic and attentional components in perception of size-at-distance. Perception \& Psychophysics, 40, 256-262.

EPSTEIN, W., LovIrTs, B. E. (1985). Automatic and attentional com- ponents in perception of shape-at-slant. Joumal of Experimental Psychology: Human Perception \& Performance, 11, 355-366.

GoGEL, W. C. (1979). The common occurrence of errors of perceived distance. Perception \& Psychophysics, 25, 2-11.

GogeL, W. C. (1980). The sensing of retinal motion. Perception \& Psychophysics, 28, 156-163.

GoGEL, W. C. (1982). Analysis of the perception of motion concomitant with a lateral motion of the head. Perception \& Psychophysics, 32, 241-250.

GoGEL, W. C. (1983). An illusory motion of a stationary target during head motions is unaffected by paradoxical retinal motion: A reply to Shebilske and Proffitt (1983). Perception \& Psychophysics, 34, 482-487.

GoGel, W. C., TIETz, J. (1973). Absolute motion parallax and the specific distance tendency. Perception \& Psychophysics, 13, 284-292.

GoGEL, W. C., \& TiETz, J. (1974). The effect of perceived distance on perceived motion. Perception \& Psychophysics, 16, $70-78$.

GOGEL, W. C., \&IETZ, J. (1977). Eye fixation and attention as modifiers of perceived distance. Perceptual \& Motor Skills, 45, 343-362.

HAY, J. C., \& SAWYER, S. (1969). Position constancy and binocular convergence. Perception \& Psychophysics, 5, 310-312.

Hochrerg, J. (1956). Perception: Toward the recovery of a definition. Psychological Review, 63, 400-405.

Hochberg, J. (1968). In the mind's eye. In R. N. Haber (Ed.), Contemporary theory and research in visual perception (pp. 309-331). New York: Holt, Rinehart \& Winston.

HoCHBERG, J. (1974). Higher order stimuli and interresponse coupling in the perception of the visual world. In R. B. McLeod \& H. L. Pick (Eds.), Perception: Essays in honor of James J. Gibson (pp. 17-39). Ithaca, NY: Cornell University Press.

Hochberg, J. (1981). On cognition in perception: Perceptual coupling and unconscious inference. Cognition, 10, 127-134.

Hochberg, J., \& Peterson, M. A. (1987). Piecemeal organization and cognitive components in object perception: Perceptually coupled responses to moving objects. Journal of Experimental Psychology: General, 116, 370-380.

HochBERG, J., \& SPIRON, J. (1985). The Ames window: Unveridical "direct" perception and not perceptual inference? [Abstract] Proceedings of Eastern Psychological Association, 56, 38.

JERISON, H. J. (1967). Apparent motion of a vista: An illusion of perspective. American Journal of Psychology, 80, 448-453.

MACH, E. (1959). The analysis of sensations (rev. 5th ed., C. M. Wi1liams, Trans.). New York: Dover. (Original work published 1895)

McNicol, D. (1972). A primer of signal detection theory. (pp. 99-119). London: George Allen \& Unwin.

Miller, G. A. (1962). Psychology: The science of mental life. New York: Harper \& Row.

OYamA, T. (1977). Analysis of causal relations in the perceptual constancies. In W. Epstein (Ed.) Stability and constancy in visual perception (pp. 183-216). New York: Wiley.

Peterson, M. A. (1984). Measures of selective components in perceptual organization. Unpublished doctoral dissertation, Columbia University, New York.

Peterson, M. A. (1986). Illusory concomitant motion in ambiguous stereograms: Evidence for nonstimulus contributions to perceptual organization. Journal of Experimental Psychology: Human Perception \& Performance, 12, 183-193.

Post, R. B., Leinowtz, H. W. (1982). The effect of convergence on the vestibulo-ocular reflex and implications for perceived movement. Vision Research, 22, 461-465.

Rogers, B., * Graham, M. (1979). Motion parallax as an independent cue for depth perception. Perception, 8, 125-134.

ShebILSKe, W. L., ProfrTt, D. R. (1981). The priority of perceived distance for perceiving motion has not been demonstrated: Critical comments on Gogel's "The sensing of retinal motion." Perception \& Psychophysics, 29, 170-172.

SHEBILSKe, W. L., \& ProffitT, D. R. (1983). Paradoxical retinal motion during head movements: Apparent motion without equivalent apparent displacement. Perception \& Psychophysics, 34, 476-481. 
SinsTEDEN, W. J. (1860). Über ein neues pseudokoptisches Bewegungsphänomen. Poggendorffs Annalen, 111, 336-339.

TODD, J. T. (1982). Visual information about rigid and nonrigid motion: A geometric analysis. Joumal of Experimental Psychology: Human Perception \& Performance, 8, 238-252.

Ullman, S. (1979). The interpretation of visual motion. Cambridge, MA: MIT Press.

voN HoRnBostel, E. M. (1922). Über optische Inversion. Psychologishe Forschung, 1, 130-156.

Wallach, H., Stanton, L., Becker, D. (1974). The compensation for movement-produced changes in object orientation. Perception \& Psychophysics, 15, 339-343.

\section{NOTES}

1. Proximal motions in and of themselves do not have direction. (The signs are assigned for purposes of summing real rotation and parallax.)
Therefore, unsigned magnitudes of the proximal motion were used in calculating these correlations.

2. Inasmuch as the range of theoretical rotations was greater than the range of the proximal rotations, correlations were recalculated on the basis of a suitably reduced range. In 7 out of 8 cases, the correlations remained significant.

3. Indeed, counterclockwise rotations as large as $42^{\prime} / \mathrm{sec}$ in the $14^{\prime} / \mathrm{sec}$ parallax condition and as large as $56^{\prime} / \mathrm{sec}$ in the $27^{\prime} / \mathrm{sec}$ parallax were rated as stationary in the front-biased condition, but not in the backbiased condition. We did not have a large enough range of clockwise rotations with which to compare these ratings.

\title{
Announcement
}

\author{
Second Conference of the International Federation of Classification Societies \\ Charlottesville, Virginia
June 27-30, 1989 \\ Charlottesville, Virgin
June 27-30, 1989
}

(Manuscript received October 20, 1986; revision accepted for publication December 7,1987 .)

The International Federation of Classification Societies (IFCS) is organizing its second conference, to be held at the University of Virginia in Charlottesville, from June 27 to June 30,1989 . This conference is devoted to the presentation of theoretical, methodological, and applied papers on classification, pattern recognition, and related methods of statistics and data analysis in the broad sense. It includes mathematical, statistical, and practical investigations in special fields of knowledge, and the interface between classification and the information sciences.

Papers are invited for the meeting. Suitable topics include: Classification, discrimination, aggregation, and clustering methods - Pattern recognition and image analysis methods • Statistical and probabilistic methods for data analysis and classification - Similarity and distance measures, data quality, and reliability - Multidimensional scaling and structure recovery methods - Consensus methods and correspondence analysis methods - Biological taxonomy, genome/molecular sequencing - Analysis and comparison of tree and graph patterns - Artificial intelligence and expert systems for classification - Classification and clustering algorithms and algorithmic aspects - Classification and clustering software for microcomputers and supercomputers - Computer graphics for classificatory problems - Practical applications in fields of biological sciences, information sciences, life sciences, mathematical sciences, medical science, and social sciences.

Please address general inquiries about the conference to IFCS-89, Department of Mathematics, University of Virginia, Charlottesville, VA 22903; (804)924-4919; Bitnet: SJT@VIRGINIA.

If you plan to present a paper, send an abstract in English, no more than 1 page in length, to Robert $F$. Ling, Chairman, IFCS-89 Program Committee, Department of Mathematical Sciences, Clemson University, Clemson, SC 29634-1907, or Bitnet: RFLING@CLEMSON). Submission of an abstract must be accompanied by a title, keywords, name(s) and institutional affiliation(s) of author(s), and the name of the contacting author for papers with multiple authors. The deadline for the submission of abstracts is January 15, 1989. 